\title{
Frontières
}

\section{Poésie et deuil}

\section{Les très riches Heures de Fernand Ouellette}

\section{Lydia Lamontagne}

Volume 19, numéro 2, printemps 2007

Penser sa mort?

URI : https://id.erudit.org/iderudit/017495ar

DOI : https://doi.org/10.7202/017495ar

Aller au sommaire du numéro

\section{Éditeur(s)}

Université du Québec à Montréal

ISSN

1180-3479 (imprimé)

1916-0976 (numérique)

Découvrir la revue

Citer cet article

Lamontagne, L. (2007). Poésie et deuil : les très riches Heures de Fernand Ouellette. Frontières, 19(2), 34-40. https://doi.org/10.7202/017495ar
Résumé de l'article

Cette étude porte sur la place qu'occupe la mort-propre dans Les heures de Fernand Ouellette. L'auteur raconte, dans une poésie haletante, les derniers moments et le décès de son père. Alors que ce dernier vit un deuil, lorsqu'il apprend que la mort s'annonce pour lui, les membres de son entourage en viennent eux aussi à considérer leur propre finitude. La communication entre le père et l'entourage laisse progressivement place au silence et l'écriture permet de reconstruire du sens là où il ne semblait plus y en avoir. Le corps devient le seul espace où se poursuit le cheminement du mourant. Cette traversée intérieure du deuil permet d'atteindre l'acceptation de la mort dans un désir de rejoindre la Lumière.
Ce document est protégé par la loi sur le droit d'auteur. L'utilisation des services d'Érudit (y compris la reproduction) est assujettie à sa politique d'utilisation que vous pouvez consulter en ligne.

https://apropos.erudit.org/fr/usagers/politique-dutilisation/ 


\section{Résumé}

Cette étude porte sur la place qu'occupe la mort-propre dans Les heures de Fernand Ouellette. L'auteur raconte, dans une poésie haletante, les derniers moments et le décès de son père. Alors que ce dernier vit un deuil, lorsqu'il apprend que la mort s'annonce pour lui, les membres de son entourage en viennent eux aussi à considérer leur propre finitude. La communication entre le père et l'entourage laisse progressivement place au silence et l'écriture permet de reconstruire du sens là où il ne semblait plus y en avoir. Le corps devient le seul espace où se poursuit le cheminement du mourant. Cette traversée intérieure du deuil permet d'atteindre l'acceptation de la mort dans un désir de rejoindre la Lumière.

Mots clés: Les heures - Fernand Ouellette - poésie - silence - corps - deuil - père.

\section{Abstract}

This study discusses perceptions of one's own death in Fernand Ouellette's Les heures, in which, in breathless poetry, he describes his father's dying moments. Confronted with his approaching death, his father mourns, and his family comes to consider the finiteness of their own life. Ouellette's writing helps him find meaning where it no longer seems to exist, as communication between father and family is gradually overtaken by silence. The dying man's body now defines the space in which he continues on his course. This voyage within makes it possible to accept death and try to reach the Light.

Keywords: Les heures - Fernand Ouellette - Poetry - Silence - The body - Mourning - Father.

\section{POÉSIE ET DEUIL Les très riches Heures de Fernand Ouellette}

\author{
Lydia Lamontagne, \\ doctorante en lettres françaises avec spécialisation en \\ études canadiennes, Université d'Ottawa.
}

La mort viendra et elle aura tes yeux cette mort qui est notre compagne [...]

O chère espérance,

ce jour-là nous saurons nous aussi que tu es la vie et que tu es le néant (Cesare Pavese, 22 mars 1950, p. 207).

Au corps fermé, qui aurait pu substituer son propre corps?

(Ouellette, 1988, p. 14)

Un parcours exceptionnel a jusqu'à présent conduit Fernand Ouellette à explorer tant l'écriture poétique, essayistique que romanesque. Il est un poète en quête de lumière et sa conception de la poésie s'avère nettement idéaliste. Pourtant, dès ses premiers ouvrages Ces anges de sang (1955) et Le soleil sous la mort (1965), l'œuvre abondante et variée de Fernand Ouellette est empreinte du thème polymorphe de la mort. En 1987, l'auteur fait paraître Les heures, un recueil dédié à son père et qui lui vaudra le prix du Gouverneur général. Selon Jean Basile de La Presse, Ouellette a écrit « avec une voix haute et grave, toujours intelligible et toujours mystérieuse » (Basile, 1987, E3), un chant dont la musique saccadée mais sans exubérance permet de saisir le temps fuyant. Freud a énoncé l'irreprésentabilité de notre propre anéantissement et le besoin de combler - pour soi - l'interrogation et le «non-savoir» (Freud, 1988a et b) qu'est la mort par sa seule représentation possible, celle de l'autre. Les heures seraient d'abord le «dialogue» intérieur d'un sujet en deuil. Elles illustreraient, ne serait-ce que par leur titre, la volonté de garder la mémoire, non pas tant des faits entourant l'agonie, mais plutôt l'hésitation du mourant et de son entourage entre le vide et la plénitude, l'attachement à la vie et le détachement, la parole et le silence. Ainsi, le «conformisme mimétique ${ }^{1}$ » (Mertens, 1996, p. 3) de l'entourage du mourant a laissé cours à une poésie où coexistent la retenue et ce dernier don de soi.

De nombreux critiques ont souligné la prose déguisée par la versification -dans le recueil. En ce sens, on remarque les nombreuses occurrences des pronoms 


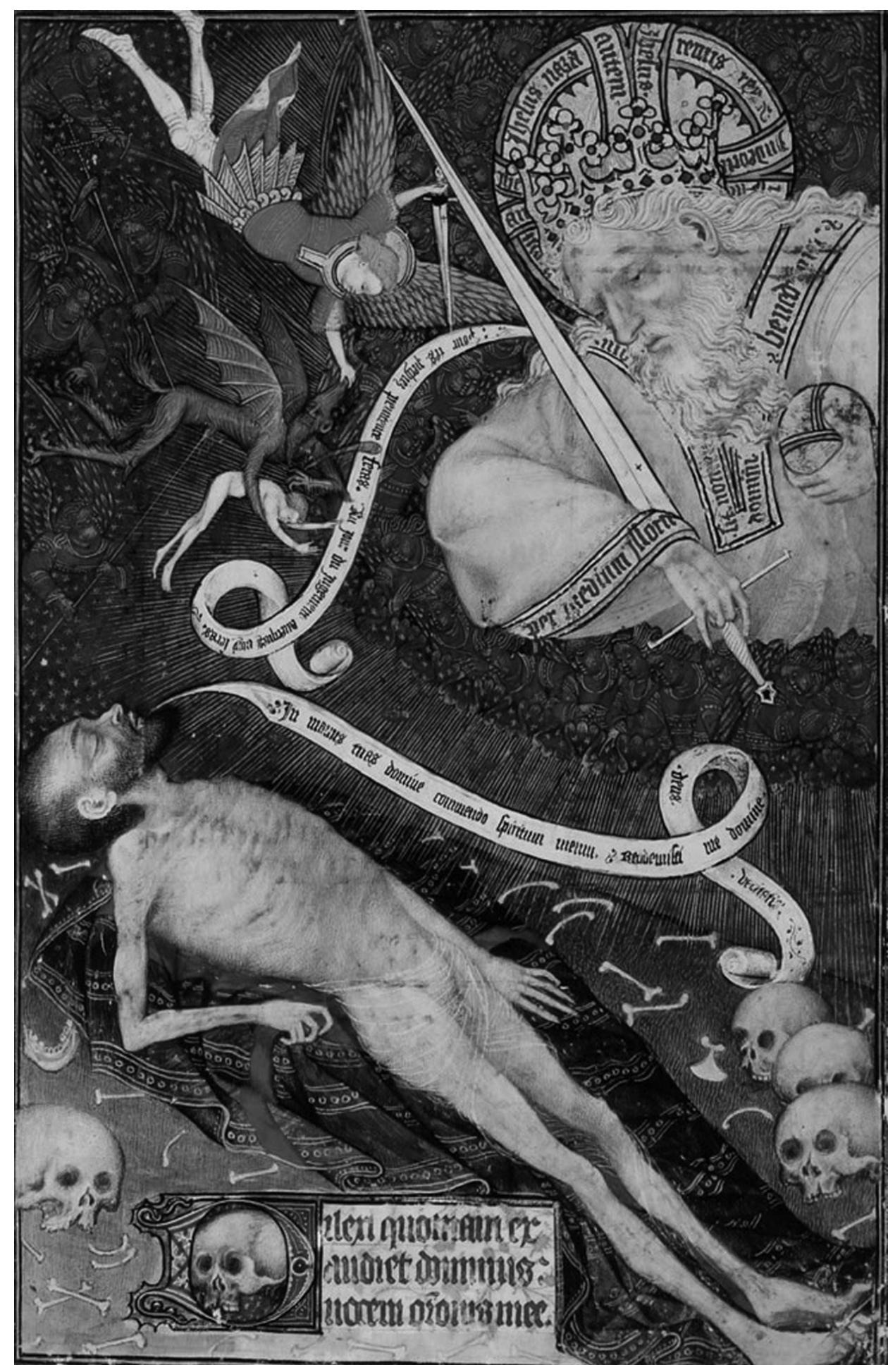

il et nous, et ce dès l'incipit: "Il était pour nous / comme une demeure» (Ouellette, 1988, p. 11). Déjà le corps en déclin, dernier point de jonction entre la vie et la mort, devient un espace à définir parce qu'il ne peut plus contenir la conscience individuelle. En somme, on peut supposer ici que le processus de création-distan-

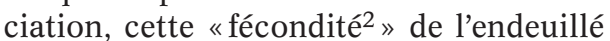
selon les termes d'Emmanuel Lévinas, aurait permis à celui qui regarde la mort de l'autre de franchir les étapes du deuil, de combler son silence et, par-dessus tout,
L'homme devant son juge (c. 1420), Maître des Heures de Rohan.

de constater que le lien père-fils est bousculé par l'annonce fatidique. Ensuite, nous verrons que le sujet endeuillé en vient à considérer sa propre perte, l'écriture permettant de reconstruire du sens là où il ne semblait plus y en avoir. Cela nous amènera finalement à considérer la mort comme un espace exigu à franchir pour accéder à l'immensité, au détachement et peut-être même à la liberté.

\section{QUELQUES CONSIDÉRATIONS FORMELLES}

Quoique Dans le sombre et Les heures aient en commun une "polarisation thématique» (l'érotisme dans le premier, la mort dans le second) et que les deux recueils aient été rédigés dans un court laps de temps (environ un mois dans les deux cas), Fernand Ouellette n'hésite pas à dire que «l'écriture elle-même [lui] paraît profondément différente dans Les heures » (cité dans Guénette, 1987, p. 5).

Joseph Bonenfant, dans un article publié une première fois en 1972, disait à propos du vers «La mort n'est-elle pas la parole?»(Ouellette, 1979, p. 251) que «le sens repose sur un rapport entre écrire et mourir» (Bonenfant, 1992, p. 113). L'extrait suivant des Heures traduit la nonsignification devant laquelle nous place le vide:

\section{Il y avait en nous}

un épuisement des sens,

une quête vaine

vers le mystère

de celui qui partait

(Ouellette, 1988, p. 49).

À la suite du décès de son père, Nicole Brossard fait débuter son recueil $\bar{A}$ tout regard par l'incipit: "Rien ne pouvait plus s'écrire»(Brossard, 1989, p. 15). De la même manière, dans l'extrait des Heures ci-dessus, les termes "une quête vaine» et «mystère » renvoient à l'inconnaissable, à la mort. La phrase de Nicole Brossard ${ }^{3}$ qui annonçait pourtant la fin de l'écriture a donné lieu à un véritable torrent de mots. L'auteure féministe explique ce déversement en disant que la perte de sens vécue lors d'un deuil entraîne une reconstruction du sens. Voilà pourquoi selon elle la littérature du deuil donne souvent lieu à de longs poèmes tels Les heures. Ces longs poèmes, fruits de la perte (au sens propre ou figuré) de l'être aimé, se rejoignent par la charge émotive qu'ils suscitent. Denise Brassard, dont la thèse de doctorat portait sur «la fonction de l'essai 
dans la démarche poétique de Fernand Ouellette », parle justement d'« anamnèse » pour expliquer la «fièvre d'écriture» et la «mémoire vivace» dont fut saisi Ouellette pendant la rédaction des Heures (Brassard, 2001, p. 344). Sept mois après le décès de son père dont il a pris soin jusqu'à la fin, Fernand Ouellette, déjà romancier, essayiste et poète québécois de renom, entreprend la rédaction de quelques poèmes aux vers succincts et aux nombreuses articulations syntaxiques. Du 23 janvier au 23 février 1986, le poète composera donc 81 poèmes, nombre correspondant à l'âge du défunt. Il commentera le nombre de poèmes en disant qu'il s'agit d'« un multiple de 9, chiffre spirituel par excellence» (Ouellette en entretien avec Gaudet, 1987, p. 20). Par ailleurs, bien qu'il soit divisé en cinq sections relatant les cinq étapes du deuil, le recueil se compose de poèmes assez rapprochés pour être lus comme un seul long poème.

Il est essentiel de noter, comme l'a fait Brassard, que Ouellette a beaucoup parlé de ce recueil, qu'il avait senti le besoin d'en «fournir certaines clés » (Brassard, 2001, p. 345). Toutefois, comme nous le verrons, il semble prudent de garder nos distances quant aux «indices » extradiégétiques de l'auteur. Interrogé par Gérald Gaudet sur l'élaboration du recueil, Ouellette dira qu'il souhaitait «donner l'illusion que poétiquement on pouvait se confronter à la mort, la regarder en face, sans tomber dans la morale, le sermon, qui a toujours nourri cette tentative» (Gaudet, 1987, p. 20). À titre de contre-exemple de son projet d'écriture, même s'il s'intéressera ultérieurement au mysticisme (à preuve les essais qu'il publiera par la suite sur le mysticisme et sur sainte Thérèse de Lisieux), Ouellette cite les Vers de la Mort rédigés à la fin du $\mathrm{XII}^{\mathrm{e}}$ siècle par le moine cistercien Hélinand de Froidmont. Dans ce texte médiéval aux allures de Danse macabre, Froidmont tente de convaincre, par la peur, les hommes de délaisser les vices du monde matériel pour préparer le départ vers la vraie Vie. Quoique la figure de la Grande Faucheuse ne soit pas explicitement présente dans Les heures, il reste que la peur de la mora certena fait partie du livre, alors que la famille et le mourant, d'abord angoissés, parviennent à surmonter la peur de l'inconnu. Ainsi n'est-il pas rare de rencontrer au fil de la lecture des passages comme le suivant, surtout dans la première partie de l'œuvre:

Nous étions pétrifiés
comme des arbres
sans feuilles,
sans sève
et sans oiseaux
(Ouellette, 1988, p. 48).
Dans le passage ci-dessus, l'entourage ne vit donc pas totalement sous le joug de la Grande Faucheuse, comme le veut un certain enseignement du christianisme (voir le livre de Jean Delumeau, Le Péché et la peur), mais il est loin d'être délié de l'angoisse de la finitude. De même, l'arbre signifie - par ses racines - l'attachement à la vie, mais nourrit la mort parce que ce symbole de vie se transforme en pierre. La victime de la mort toute-puissante est, elle aussi, rongée par la peur:

\section{Dans nos bras,}

noué et craintif,

il se laissait prendre.

Mais les peurs mâchées,

de pensée en souvenir,

savaient le dissoudre

(Ouellette, 1988, p. 18).

Plusieurs termes dans cet extrait soulignent non plus la «monumentalisation» comme représentation de l'angoisse du danger de mort mais plutôt la dissolution. Les adjectifs «noué et craintif» au singulier s'accordent avec le sujet «il» et non avec l'entourage représenté par l'adjectif possessif «nos ». Quant à l'adjectif «mâchées », il traduit les dommages causés par les craintes. Quoique cette désagrégation qui deviendra plus tard émancipation post mortem ne soit jamais remise en question par le «nous» ou le mourant (comme c'est le cas dans la liturgie qui utilise la peur), le recueil dépeint tout de même la nécessité de cheminer vers l'acceptation de la non-existence, un cheminement essentiel pour atteindre la Lumière. Poète dont la quête mystique sera plus évidente $a$ posteriori, selon Denise Brassard (Brassard, 2001, p. 344), Ouellette se rapproche malgré tout de la littérature médiévale. En effet, l'approche du mourir qui est décrite dans le recueil s'apparente à la «mort apprivoisée » dont parle l'historien des mentalités Philippe Ariès (Ariès, 1977, p. 13-36) à propos de la première partie du Moyen Âge. À titre indicatif, on trouve quelques allusions aux «orant[s]» (Ouellette, 1988 , p. 49 et 81), ces personnages représentés dans l'attitude de la prière propre à l'architecture chrétienne primitive (Mâle, 1949). On trouve aussi les termes «l'opulence/des ténèbres »(Ouellette, 1988, p. 19), les «chimères»(Ouellette, 1988, p. 26) et «l'entre-monde... »(Ouellette, 1988, p. 20). Par conséquent, exhortation et frayeur en moins, le recueil de Ouellette n'est pas étranger, y compris dans son titre même, à une tradition qui a connu son apogée à la fin du Moyen Âge : le livre d'heures. Interrogé à ce sujet, Ouellette dit ne jamais avoir pensé à ces prières de l'office comme l'a fait Henry Bauchau dans une œuvre (Guénette, 1987, p. 61). Pourtant, le format étroit du petit livre de Ouellette dans sa première édition rappelle, à lui seul, la typographie des poèmes principalement formés de vers très courts (on dénote notamment plusieurs bisyllabes) dont la musique «donne le sentiment d'un temps compté» (Melançon, 1987, p. 4). Ces considérations formelles s'apparentent aux contraintes imposées par la miniature associée aux livres d'heures. Il convient de rappeler que ces ouvrages précieux représentaient en huit sections les 24 heures de la Vierge et se terminaient habituellement par l'office des morts. (Notons aussi que les illustrations de ces livres de dévotion personnelle faisaient parfois référence à la biographie de leur riche propriétaire.) De manière similaire, Ouellette décrit dans une forme très hachurée les dernières heures de son père qu'il divise en cinq étapes de longueur inégale. Nous les avons intitulées, dans l'ordre: l'annonce, le deuil anthume et posthume, les derniers instants, la dépouille et l'au-delà, et finalement l'acceptation et l'anticipation de la mort. Les cinq étapes du deuil auxquelles les études sur la mort font souvent référence se trouvent en porteà-faux par rapport à ces sections poétiques : le déni, la colère, la négociation, la dépression, l'acceptation. Comme c'est souvent le cas dans le deuil tel qu'il est vécu hors du texte, certaines étapes se juxtaposent dans Les heures; on assiste parfois à une régression, et ce jusqu'à la fin du livre qui ouvre pourtant sur l'éternité.

\section{ENTRE PÈRE ET FILS: LA MORT}

À propos de l'annonce fatidique, le poète dit ceci : "À partir du moment où je vois, je sais qu'il sait, je ne le laisse plus. Je vais à l'hôpital. Je deviens à la maison son infirmier»(Gaudet, 1987, p. 20). Or, Ouellette ne mentionne jamais explicitement sa famille ou l'entourage du défunt, ni dans son recueil ni en entrevue. Un passage des Heures laisse croire à un "nous » qui désignerait plusieurs locuteurs :

Nous lui adressions

des pensées,

chacun depuis

sa solitude

(Ouellette, 1988, p. 103).

Le «nous» pourrait inclure tout un chacun, y compris le lecteur qui vit un décès en différé ou qui entrevoit sa propre finitude. Les pensées représentent alors les paroles, les poèmes adressés au moribond qui s'approche du secret de la mort. Les nombreux points d'interrogation (trente-sept au total) traduisent des questions posées à celui qui percera le secret du mourir. Ces questions portant sur l'inconnaissable montrent l'entrecroisement de la réflexion sur la mort d'autrui et la mort-propre. Elles touchent le lecteur qui devient sensible à cette tentative de compréhension de Thanatos. De plus, elles révèlent l'essayiste dans l'écriture poétique. 
Le recueil de Ouellette raconte le cheminement de la mort annoncée jusqu'au moment où seul "l'objet funèbre» (Ouellette, 1988, p. 77) du père subsiste. À l'exception de la dédicace "À mon père", ce dernier est toujours mentionné à la troisième personne du singulier comme si l'auteur souhaitait se distancer d'un sujet avec lequel il a pourtant été étroitement lié. Ainsi, la mort s'introduit dans le corps de celui qui doit dès lors se préparer à quitter le monde de la parole pour celui du regard, puis du silence. Déjà l'annonce de la terrible nouvelle empêche le dialogue:

Il n'a pas eu le temps

de se défier

du langage.

Des mots implacables

l'ont muré

(Ouellette, 1988, p. 16).

Ces vers justifient la narration par la volonté de raconter l'inénarrable pour celui qui le vit. Il pourrait s'agir d'écrire la mort de l'autre pour la faire sienne, pour imaginer sa mort. Ces vers dépeignent
Paul Chanel Malenfant nomme «la lente monotonie répétitive du temps de l'agonie du père » (Malenfant, 2001, p. 252). Celuici est représenté par le sujet écrivant dans l'extrait ci-dessous :

Le temps, le temps,

dans les éclaircies de conscience,

avait des pas de plomb

(Ouellette, 1988, p. 21).

Il y aurait donc deux deuils dépeints dans ce livre, celui du «nous» qui s'accroche au sujet en train de mourir, à sa dépouille, puis à son souvenir et celui du «il» qui se déroule plus lentement, traduisant ainsi un rapport au temps tout autre. Il y aurait deux postures du deuil et elles correspondraient à celles remarquées par Nicole Brossard: l'une définie par la vitesse, voire la violence, l'autre se caractérisant par la lenteur et la langueur.

Quoique le trajet soit narré en accéléré par le «nous», on peut affirmer que l'entourage contribue aussi à ralentir le rapport au temps du sujet mourant et, par conséquent, son détachement de la vie.

\section{LE MUTISME DU PÈRE ET LE NON-SAVOIR DE LA MORT} ONT PERMIS L'OUVERTURE À UNE POÉSIE OÙ L'AUTRE EST INTÉGRÉ EN SOI, OÙ IL DEVIENT SOI.

l'emprisonnement que constitue l'attente de la fin imminente. Le poète sait qu'il vole l'autre, qu'il le dépouille puisque le moribond ne peut simultanément raconter sa mort et la vivre:

En somme,

chacun de nous,

avec son être disjoint,

et mal initié à l'obscur,

au dédale,

se préparait

à lui voler

sa mort

(Ouellette, 1988, p. 24-25).

L'entourage tente de percer pour soi «le secret de l'agonie» (Ouellette, 1988, p. 19), cherche à lire et à interpréter les derniers instants qui se déroulent lentement à cause de l'attention portée aux détails, et ce en dépit du ton haletant du livre. Ainsi, il y a double temporalité dans Les heures. D'un côté se trouve représentée l'urgence de saisir tous les instants (on y trouve la vitesse qui correspond au temps de la rédaction) de peur de lâcher prise et de laisser partir le mourant. De l'autre s'écoule le temps vécu par le moribond au fur et à mesure que sa présence au monde diminue doucement. C'est ce que trépas coexistent. Ni centrée sur le parricide psychanalytiquement associé à la mort du père lorsqu'il est question du fils ni sur un quelconque jeu de pouvoir, la figure du père des Heures ne se compare donc pas à celle remarquée dans la littérature québécoise par François Ouellet ${ }^{4}$. Toutefois, nous sommes d'accord avec ce dernier pour dire que «le père est à la fois ce qui structure intérieurement le sujet et le signe qui le dépasse » (Ouellet, 2003, p. 99). En ce sens, le pronom «il» constitue un élément narratif important des Heures puisqu'il confirme non pas le besoin de compenser la perte de l'objet désiré, mais celui de vivre, par l'écriture, l'abandon nécessaire à l'aboutissement du deuil. Par conséquent, la poésie permet d'intérioriser la figure du père, d'où les nombreuses images du corps qui se referme dans le processus du mourir.

En dépit de cette écriture de l'intime et l'emploi du genre masculin, le pronom «il » rend universelle l'expérience du mourir :

Il demeurait

devant nous

frappé de plein fouet

par sa lucidité dévoilante

(Ouellette, 1988, p. 13).

En effet, le pronom souvent en position anaphorique focalise la narration sur le moribond, combattant, puis acceptant son sort qui est celui de tous les hommes. À cela, ajoutons l'emploi quasi exclusif de l'imparfait qui transforme cette poésie prosodique en une suite de tableaux (voire des scènes) illustrant les craintes, les interrogations et les étapes préparatoires au mourir. Tout cela ressemble aux Artes moriendi de la fin du Moyen Âge. Le livre va bien au-delà d'un hommage au père livré par son fils ou d'une tentative d'en conserver la mémoire, il s'apparente à un guide du bien mourir.

Un vers rompt la dénomination du père avec le pronom «il». Il s'agit du vers :

«Tu ne dois pas partir»

(Ouellette, 1988, p. 87)

Le pronom «tu» implique un «je», alors que les guillemets témoignent d'une tentative de dialogue. Le pronom à la deuxième personne du singulier ainsi que les guillemets qui encadrent le vers soulignent l'importance de ce passage clé de l'œuvre. C'est un véritable cri du cœur. Le verbe est à l'indicatif présent plutôt qu'au conditionnel, ce qui permet au fils d'exprimer une certaine colère - l'une des étapes du deuil. Le locuteur s'adresse ici directement à son allocutaire, son père. Nous pouvons penser que cet unique tutoiement exprime plus clairement la résistance à l'abandon de la position libidinale dont parle Freud dans «Deuil et mélancolie» 
et qui sous-tend l'angoisse exprimée par l'entourage dans Les heures. Luc Bouvier, dans son analyse du «je» dans la poésie de Jacques Brault, rappelle que le «il » peut être un dédoublement $\mathrm{du}$ «je» (Bouvier, 1988, p. 19). En ce sens, la mort dont il est ici question pourrait aussi bien être celle que le «je» anticipe pour lui-même. À la fin du livre, il est directement question de la mort $\mathrm{du}$ "nous». Or, les formes dites plurielles de «je» et «tu» désignent plus qu'une simple augmentation du nombre ou la formule de politesse dans le cas du «vous» : il s'agit d'une «amplification de la personne» (Maingueneau, 2000, p. 7). Une fois le chemin ouvert par le sujet devenu une des «voix lumineuses », le «nous», soit le «je » et le lecteur, peut lui aussi franchir la dernière étape du deuil, l'acceptation de sa propre mort:

Mais nous retrouverons

nos morts.

Nous les reconnaîtrons

à leurs voix lumineuses.

$[\ldots]$

Nous ne pouvons

plus reculer

(Ouellette, 1988, p. 109-110).

Certes, les deux derniers vers indiquent que la quête reste vaine et la mort, une énigme. Mais le message en est un d'espoir et de lumière. Puisque le «nous » a survécu à la mort de l'être cher, il pourra vivre la sienne sans crainte.

Cette pratique énonciative qui emploie le «nous» n'est pas coutumière dans la poésie de Ouellette. En 1995, dans la préface de la réédition de Soleil sous la mort, François Dumont évoque la distance qui habite le jeune Ouellette, distance que cherchera à conquérir le poète «tout au long de son apprentissage jamais terminé » (Dumont, 1995, p. 7). Le critique interprète une section du Soleil sous la mort écrite au "nous» comme une expression de la collectivité par opposition au «on» incarnant la force de l'aliénation (Dumont, 1995, p. 9). Nonobstant une telle lecture et certains essais virulents de Ouellette sur la langue française, Laurent Mailhot et Pierre Nepveu croient que cette écriture « porte à peine la trace du lyrisme national des années soixante» (Mailhot et Nepveu, 1996, p. 344). Ainsi, la parole collective des Heures n'est clairement pas attribuable à la «poésie du pays ». Elle vise plutôt à rejoindre une humanité du même ordre que celle exprimée par Borges dans une épigraphe du Soleil sous la mort: «Mon humanité, c'est de sentir que nous sommes les voix d'une même misère » (Ouellette, 1995, p. 69). Par conséquent, il semble juste d'affirmer que l'intention de l'auteur dans l'utilisation des pronoms «nous» et «il» était de rendre universelle l'expérience intime de la mortalité humaine. Aux dires du poète: «Écrire, c'est se mettre en relation avec les autres et les relier ainsi à sa propre recherche» (Gaudet, 1987, p. 19). Au-delà de l'omniprésence du père dans Les heures, nous sommes de l'avis de Gaudet qui croit que «Les heures est le récit d'un homme qui se penche sur sa propre mort»(Gaudet, 1987, p. 16). C'est la raison pour laquelle le "nous» occupera progressivement plus de place dans Les heures, si bien qu'il parvient par la réflexion post mortem à dépasser le deuil, à lâcher prise, pour envisager la renaissance du soi.

\section{L'ESPACE DE LA MORT: DE L'EXIGU À L'IMMENSE}

L'incipit met bien en évidence l'importance du corps par une comparaison entre l'espace sécurisant de la maison et la figure du père:

Il était pour nous

comme une demeure

sans limites,

bruissante de rumeurs

rassurantes.

Au moindre vent,

nous nous glissions

sous ses pensées

(Ouellette, 1988, p. 11).

Selon Gaston Bachelard, la maison est un lieu relié à l'être du dedans, à l'intime. Le père de la critique thématique parle de «la maternité de la maison» où se vit la "plénitude première de l'être » (Bachelard, 2001, p. 27). Les nombreuses métaphores du père-arbre confirment l'existence d'un espace où il est possible pour l'être aimé de loger son amour. Dans l'extrait ci-dessus, les reprises phoniques de la lettre «s» se concentrent pour suggérer un souffle réconfortant, une musique « rassurante[s] » par opposition au silence dissociatif et déstabilisant de la mort. L'allitération ainsi que le vers «bruissante de rumeurs» annoncent l'intention du poète de rompre le silence. Son père lui parlant très peu (selon Gaudet, 1987, p. 21), il n'est pas étonnant que les champs sémantiques de la solitude et du silence traversent l'œuvre ouellettienne:

Tant qu'il parlait,

même bas,

il restait lié

au silence qui pense

$[\ldots]$

N'avait-il pas toujours été,

face à nous,

l'abîme des énigmes?

(Ouellette, 1988, p. 39)

À l'instar des pensées qui rassuraient la famille dans le passage précédent, c'est maintenant le silence qui fait penser à la mort, et ce, même lorsque le mourant murmure. Pourtant le père était associé au nondit avant la venue de la Grande Faucheuse. Homme réservé, il était déjà une énigme pour la famille, une énigme rassurante parce que familière, tout au contraire de la mort. L'image du père «habitable» se perd peu à peu tandis que «l'interrogation totale de la mort»(Brochu, 1987, p. 166) se mêle au silence du père dont le corps, tranquillement pour le mourant et rapidement pour l'entourage, s'éloigne du monde des vivants :

Il y avait près de nous,

en lui,

l'extase d'une imagination

horrifiante,

l'intimité d'un silence

bientôt infranchissable

(Ouellette, 1988, p. 19).

Le silence devient un mur entre le mourant et son fils. Il témoigne de la dissociation du moi (Guiomar, 1988, p. 96) pendant que l'âme glisse hors du corps. Ce mutisme affecte le monde des vivants car il réduit le dialogue et l'amour filial à la tâche de la mémoire "oublieuse» (Ouellette, 1988 , p. 48). En parallèle du souvenir qui s'efface, le corps disparaît:

Par-delà le séisme

muet du corps oublieux

de lui-même

et des lumières,

l'intimité entre nous

se désagrégeait.

(Ouellette, 1988, p. 48).

Encore une fois, notons l'association entre l'agonie et la dissolution. L'être tout comme le corps sont sujets à une décomposition symbolique. Toutefois, suivant les Principes d'une esthétique de la mort de Michel Guiomar, on peut affirmer que Les heures ne tomberaient pas dans le lugubre, c'est-à-dire dans un certain goût pour la mort (Guiomar, 1988, p. 173). À preuve, l'euphémisation des dommages du cancer (ce «séisme muet») où le corps «s'oublie» comme si le biologique avait sa propre conscience. Pierre Nepveu s'est penché sur la thématique du silence dans l'œuvre ouellettienne précédant Les heures. Selon lui, «le silence du locuteur est ambigu: il signifie autant l'inaptitude à parler que la disponibilité à une "autre" parole, ou à toutes les paroles» (Nepveu, 1979, p. 11). Le mutisme du père et le non-savoir de la mort ont permis l'ouverture à une poésie où l'autre est intégré en soi, où il devient soi. Le silence et l'énigme ont laissé surgir, par la voix de l'autre spectateur de la mort, une image lumineuse de la traversée ultime : 
La vie nous tient

jusqu'au silence.

Le périple commence

toujours par l'abîme

(Ouellette, 1988, p. 110).

L'abîme qu'était le père est maintenant celui de la mort. Le détachement nécessaire à l'acceptation de la mort se produit après avoir sondé les profondeurs, un peu à la manière du Tombeau des rois d'Anne Hébert. La lumière jaillit donc des cendres. Fernand Ouellette a montré «le soleil sous la mort», pour reprendre le titre du recueil paru en 1965. Si écrire, c'est substituer à l'état éphémère du corps une «trace»(Ouellette, 1988, p. 61) qui se compare à la pierre tombale comme l'exprime Michel Serres dans Statues (Serres, 1988, p. 96), écrire la mort de l'autre constitue la seule mise en scène possible de notre propre fin. À mesure que le corps rejoint l'inanimé, la distance entre le mourant et la famille se creuse. Étonnamment, on dénote alors une «monumentalisation» du locuteur:

Il était là si près

et nous si loin,

pétrifiés

d'impuissance

(Ouellette, 1988, p. 50).

Dans cet espace où l'âme est peu à peu expulsée, le «nous » en vient aussi à adopter une posture de fermeture. Il se perçoit comme une statue funéraire, un objet sans vie tel l'«orant»(Ouellette, 1988, p. 49). Freud ne disait-il pas que la littérature nous réconcilie avec la mort parce qu'elle nous offre une pluralité de vies où il est possible de mourir par identification à un héros tout en restant à l'abri (Freud, 1988a, « Notre rapport à la mort»)? Représenter la mort de l'être cher, c'est donc tenter, selon le père de la psychanalyse, de vivre la mort pour apaiser l'angoisse de notre anéantissement:

\section{On tirait}

nos racines.

Au corps fermé,

qui aurait pu substituer

son propre corps?

(Ouellette, 1988, p. 14).

Force envahissante, la mort tout en réclamant son dû, entraîne l'entourage dans la chute. Elle se présente plus macabre encore lorsqu'elle est personnifiée:

\footnotetext{
Qu'était cette mort

qui l'avait pris

en charge?

[...]

Elle allait en lui

$[\ldots]$

Elle avait tout

d'une force

parfaitement

silencieuse
}

(Ouellette, 1988, p. 57).
Le silence devient lourd, il devient fort lorsqu'il s'appelle la mort. L'envahisseur n'est pas discret dans cet extrait. Il prend d'assaut l'espace corporel. Dans le couple père-fils, la Mort est la seule présence féminine, le «elle» à la puissance destructrice. Ensorceleuse, elle n'est pas sans rappeler Les Vers de la Mort:

Mort est la main qui tout agrippe;

Elle garde tout ce qu'elle accroche

Mort taille pour tous un manteau de

tissu sombre

(Froidmont, 1983, poème XXXI).

Celle-ci accomplit sans bruit son activité nécrophage, épousant du même coup le silence du père et laissant le «nous » sans réponse ni explication quant à la mort de l'autre en soi. Bien que la personnification soit associée au macabre, le poète se garde bien de déployer tout l'arsenal qu'utilisait Froidmont. En effet, à l'exception du terme «osseux», les ravages de la maladie sont subtilement décrits :

\section{Progressait \\ la calcination entière \\ de tout ce qui l'avait fait \\ mortel \\ (Ouellette, 1988, p. 51).}

L'image du phénix surplombe cette description lorsqu'elle est lue dans le contexte d'une libération de l'instance mortifère. Emprisonné par la fatalité, le corps se replie sur soi pour s'ouvrir à l'immensité intérieure. Ce périple est défini comme une traversée "dans sa barque glissante» (Ouellette, 1988, p. 23) tout comme celle qui se trouve dans le Livre des morts des anciens Égyptiens. Le tout se déroule dans le soi, dans un emboîtement d'espaces à partir du corps: les enfants dans le père, la mort dans le père, le mystère dans la mort. Le deuil se vit lui aussi tel un enchâssement: les enfants ont intégré en eux la figure du père, à l'intérieur de celle-ci se trouve maintenant la mort prochaine, et finalement cette dernière renvoie à la finitude à venir qui attend l'entourage, et par extension, tous les hommes. Cela dit, il suffit de lire l'extrait suivant pour comprendre que le corps meurt en emportant avec lui le mystère de la mort:

Son corps

ne s'ouvrait plus.

[...]

Il s'était tourné

vers l'intérieur...

Pour le vrai départ.

Le dedans inviolable

était le seul espace

du passage,

ou l'infini

de la dérive

(Ouellette, 1988, p. 32).
Le silence de l'absence à venir et les pensées évanescentes du père imaginées par le fils donnent l'impression d'une traversée vers la mort qui a l'apparence d'une dérive, d'une étendue. Contrairement à l'emmurement de la mort dans le corps, l'âme passe du dedans au dehors. Elle semble se dissoudre, se perdre dans l'infini. Elle vit, pour reprendre les mots de Bachelard, «la conscience d'agrandissement» (Bachelard, 2001, p. 169). De même que la mort fait son chemin dans l'éphémère et rompt par sa présence l'espace de communication avec le père, le locuteur se meurt:

De plus en plus vulnérable,

notre présence

s'évanouissait

contre lui

(Ouellette, 1988, p. 14).

Nous assistons donc à un véritable retournement du rapport à la mort entre le soi et l'autre. De cette quête du secret insaisissable, le locuteur vit une renaissance qui pourrait bien se résumer à cette courte phrase de Bachelard: "L'immensité est en nous»(Bachelard, 2001, p. 169). Le renversement ultime, celui de l'enfant et du parent lors de son dernier souffle, a été décrit par le poète comme suit: "Chose étrange, quand je le tenais dans mes bras, à la fin, j'avais l'impression qu'il était devenu mon enfant» (cité dans Gaudet, 1987, p. 20). Véritable don, la mort de l'autre ouvre à l'immensité celui qui sait dépasser la perte d'une partie de soi. C'est sans doute ce que veut dire le poète lorsqu'il parle de l'«étoilement»(Ouellette, 1988, p. 15). La reproduction en page de couverture du célèbre tableau de Paul-Émile Borduas, «L'étoile noire », vient confirmer la beauté de l'inversion entre le dedans et le dehors, entre la vie et la mort, alors que les étoiles s'éloignent à l'infini.

En lisant Les heures, nous comprenons que l'intime peut rejoindre l'universel, que la mort mise à nu peut susciter, au-delà de l'angoisse tant de beauté. Faire de la mort de l'autre un poème ne fut certes pas chose facile. En ce sens, le choix des pronoms « il » et «nous» s'explique, selon nous, par une volonté de se distancer de l'«objet funèbre », à tort méprisé et difficile à accepter, pour laisser place à la réflexion sur la mort-propre. Ouellette a montré combien la retraversée des derniers moments vécus en compagnie de son père lui a permis d'atteindre, par la poésie, une meilleure compréhension de la Grande Faucheuse. Le corps agonisant s'est avéré un espace où en plus d'envisager la non-existence de l'autre, la représentation de la mort de soi fut envisageable. L'identification avec autrui (être cher ou héros de fiction) nous a permis d'atteindre, ou à tout le moins 
d'approcher cette interrogation fondamentale, ce thème qui après tout n'est que béance. Le silence du père a traduit l'isolement d'abord volontaire, puis involontaire (lorsque la maladie progresse) du mourant et la progression «intérieure» de sa propre démarche vers la mort. Fernand Ouellette a tenté de briser le lourd silence de la mort par la poésie.

\section{Bibliographie}

ARIÈS, Ph. (1977). L'homme devant la mort, tomes 1 et 2, Paris, seuil.

BACHELARD, G. (2001). La poétique de l'espace, Paris, Presses universitaires de France.

BASILE, J. (1987). «Une poésie grave, sans horreur ni effroi», La Presse, 11 juillet, p. E3.

BOUVIER, L. (1988). "Je» et son histoire: l'analyse des personnages dans la poésie de Jacques Brault, Ottawa, Les Éditions David.

BRASSARD, D. (2001). «La fonction de l'essai dans la démarche poétique de Fernand Ouellette », diplôme d'études supérieures en littérature française, présenté à la Faculté des arts de l'Université McGill.

BROCHU, A. (1987). «L'interrogation totale de la mort », dans Voix et images, vol. XIII, $\mathrm{n}^{\mathrm{0}}$ 1, p. 165-174.

BROSSARD, N. (1989). À tout regard, Montréal, Bibliothèque québécoise.

DELUMEAU, J. (1983). Le péché et la peur, Paris, Fayard.

DUMONT, F. (1995). «L'apprentissage de la distance », préface de la réédition du Soleil sous la mort, Montréal, Typo.

FREUD, S. (1988a). "Notre rapport à la mort», dans Euvres complètes, vol. XIII, Paris, Presses universitaires de France, p. $142-155$.

FREUD, S. (1988b). «Deuil et mélancolie», dans Euvres complètes, vol. XIII, Paris, Presses universitaires de France, p. 259-278.

FROIDMONT, H. (1983). Les vers de la mort, texte repris et traduit par Michel Boyer et Monique Santucci, Paris, Librairie Honoré Champion.

GAGNON, F. (1988). Paul-Émile Borduas, Montréal, Le Musée des beaux-arts de Montréal.

GAUDET, G. (1987). "Le corps vibrant de désir ", dans Lettres québécoises, n ${ }^{\circ} 44$, p. 16-21.

GUÉNETTE, D. (1987). «Les Heures de Fernand Ouellette», dans Nos livres, p. 5, 60-61.

GUIOMAR, M. (1988). Principes d'une esthétique de la mort, Paris, José Corti.

MAINGUENEAU, D. (2000). Éléments de linguistique pour le texte littéraire, Paris, Nathan Université.

MÂLE, É. (1949), L'art religieux de la fin du Moyen Âge en France, Paris, Librairie Armand Colin.
MALENFANT, P. C. (2001), «Écrire comme mourir: tombeau des mots ", dans Voix et images, vol. XXVI, n ${ }^{\circ} 2$ (77), hiver, p. 247-263.

MELANÇON, R. (1987). "Dans l'attention extrême », dans Spirale, no 74, p. 4.

MERTENS, P. (1996). "Une mort récitée: La narrativité dans les Heures de Fernand Ouellette», mémoire présenté à la Faculté des études supérieures de l'Université Laval.

NEPVEU, P. (1979). Les mots à l'écoute: poésie et silence chez Fernand Ouellette, Gaston Miron et Paul-Marie Lapointe, Québec, Presses de l'Université Laval.

OUELLET, F. (2003). Passer au rang de père: Identité sociohistorique et littéraire au Québec, Québec, Éditions Nota bene.

OUELLETTE, F. (1995). Le soleil sous la mort, Montréal, l'Hexagone.

OUELLETTE, F. (1988). Les heures, Montréal, l'Hexagone.

OUElletTe, F. (1979). Poésie: poèmes 1953-1971, Montréal, l'Hexagone.

PAVESE, C. (2002). Travailler fatigue. La mort viendra et elle aura tes yeux. Poésies variées, trad. de l'italien par Gilles de Van, Paris, Gallimard.

SERRES, M. (1987). Statues: le second livre des fondations, Paris, Éditions François Bourin.

\section{Notes}

1. Petra Mertens emprunte cette expression à Thomas H. Macho, Todesmetaphern, Frankfurt am Main, Suhrkamp, 1987, p. 7-8.

2. Denise Brassard se réfère au concept d'Emmanuel Lévinas en ces termes : «La fécondité (qui s'applique ici tout aussi bien à l'artiste qu'à la relation père-fils) permet une distance avec l'être et avec le présent, lequel, sous l'effet de la maturation ou du vieillissement, est perçu comme une limitation de l'être, une fermeture des possibles, bref des "occasions perdues" que la mémoire ne saurait rendre " (Brassard, 2001, p. 377). La fécondité se nourrit de la lutte contre l'oubli et cherche à compenser l'absence par l'œuvre créée.

3. Ces propos sont tirés de la table ronde "L'écriture du deuil dans la poésie québécoise contemporaine, rencontre avec Nicole Brossard, Denise Desautels, Louise Dupré, Stéphane Martelly organisée par Catherine Mavrikakis le 12 décembre 2006 dans le cadre du séminaire FRA 6260: «Temps prophétiques, mémoires endeuillées » et du groupe de recherche-création "Thanatographie, spectralité, événementialité ». Je résume les propos tels que je les ai entendus.

4. Dans Les heures, il n'est jamais question pour le fils de prendre la place du père, comme le montre François Ouellet dans son étude sociohistorique et littéraire de la figure du père, parce que c'est sous le signe de l'amour parental, voire maternel que se définit le lien père-fils. 